\title{
First HARPSpol discoveries of magnetic fields in massive stars ${ }^{\star}$
}

\author{
E. Alecian ${ }^{1}$, O. Kochukhov ${ }^{2}$, C. Neiner ${ }^{1}$, G. A. Wade ${ }^{3}$, B. de Batz ${ }^{1}$, H. Henrichs ${ }^{4}$, J. H. Grunhut ${ }^{3}$, \\ J.-C. Bouret ${ }^{5}$, M. Briquet ${ }^{6,1,8}$, M. Gagne ${ }^{7}$, Y. Naze ${ }^{8}$, M. E. Oksala ${ }^{9}$, T. Rivinius ${ }^{10}$, \\ R. H. D. Townsend ${ }^{11}$, N. R. Walborn ${ }^{12}$, W. Weiss ${ }^{13}$, and the MiMeS collaboration \\ ${ }^{1}$ LESIA-Observatoire de Paris, CNRS, UPMC Univ., Univ. Paris-Diderot, 5 place Jules Janssen, 92195 Meudon Principal Cedex, \\ France \\ e-mail: evelyne.alecian@obspm.fr \\ 2 Department of Physics and Astronomy, Uppsala University, Box 516, 75120 Uppsala, Sweden \\ 3 Dept. of Physics, Royal Military College of Canada, PO Box 17000, Stn Forces, Kingston K7K 7B4, Canada \\ 4 Astronomical Institute Anton Pannekoek, University of Amsterdam, Science Park 904, 1098XH Amsterdam, The Netherlands \\ 5 Laboratoire d'Astrophysique de Marseille, Traverse du Siphon, BP8-13376, Marseille Cedex 12, France \\ ${ }^{6}$ Instituut voor Sterrenkunde, Katholieke Universiteit Leuven, Celestijnenlaan 200 D, 3001 Leuven, Belgium \\ 7 Department of Geology and Astronomy, West Chester University, West Chester, PA 19383, USA \\ 8 FNRS-Institut d'Astrophysique et de Géophysique, Université de Liège, Allée du 6 Août 17, Bat B5c, 4000 Liège, Belgium \\ 9 Department of Physics and Astronomy, University of Delaware, Newark, DE 19716, USA \\ 10 European Organisation for Astronomical Research in the Southern Hemisphere, Casilla 19001, Santiago 19, Chile \\ 11 Dept. of Astronomy, University of Wisconsin-Madison, 475 N. Charter Street, Madison WI 53706-1582, USA \\ 12 Space Telescope Science Institute, 3700 San Martin Drive, Baltimore, MD 21218, USA \\ 13 Institut für Astronomie, Universität Wien, Türkenschanzstrasse 17, 1180 Wien, Austria
}

Received 28 October 2011 / Accepted 10 November 2011

\section{ABSTRACT}

\begin{abstract}
In the framework of the Magnetism in Massive Stars (MiMeS) project, a HARPSpol Large Program at the 3.6m-ESO telescope has recently started to collect high-resolution spectropolarimetric data of a large number of Southern massive OB stars in the field of the Galaxy and in many young clusters and associations. We report on the first discoveries of magnetic fields in two massive stars with HARPSpol - HD 130807 and HD 122451, and confirm the presence of a magnetic field at the surface of HD 105382 that was previously observed with a low spectral resolution device. The longitudinal magnetic field measurements strongly vary for HD 130807 from $\sim-100 \mathrm{G}$ to $\sim 700 \mathrm{G}$. Those of HD 122451 and HD 105382 are less variable with values ranging from $\sim-40$ to $-80 \mathrm{G}$, and from $\sim-300$ to $-600 \mathrm{G}$, respectively. The discovery and confirmation of three new magnetic massive stars, including at least two He-weak stars, is an important contribution to one of MiMeS objectives: the understanding of the origin of magnetic fields in massive stars and their impact on stellar structure and evolution.
\end{abstract}

Key words. stars: massive - stars: magnetic field - stars: chemically peculiar - stars: individual: HD 122451 - stars: individual: HD 105382 - stars: individual: HD 130807

\section{Introduction}

$\mathrm{MiMeS}^{1}$ (Magnetism in Massive Stars) is a broad collaboration that aims to address many questions concerning the magnetism of massive stars. One goal in particular is to determine the global magnetic properties of massive stars with the help of Large Programs (LP) that have been allocated to the high-efficiency high-resolution spectropolarimeters ESPaDOnS (Canada France Hawaii Telescope, Hawaii) and Narval (Telescope Bernard Lyot, France). These programs aim to observe about 200 massive OB field stars (the survey component or SC), to search for magnetic fields, confirm those previously suspected, and derive statistical properties (Wade et al. 2009; Grunhut et al. 2011b). They also aim to intensely observe about 30 already known magnetic massive stars (the targeted component or TC) to map their surface magnetic fields in detail. This smaller sample of stars is dedicated to the study of the interplay of magnetic fields with the

* Based on observations collected at the European Southern Observatory, Chile (Program ID 187.D-0917).

${ }^{1}$ http://www . physics. queensu.ca/ wade/mimes stellar structure, environment, and evolution at high mass (e.g. Grunhut et al. 2009; Oksala et al. 2011).

In 2010, the polarimeter HARPSpol was commissioned at the $3.6 \mathrm{~m}$-ESO telescope (La Silla, Chile). For the first time, we now can access the Southern Hemisphere with a data quality similar to ESPaDOnS and Narval. Therefore, a Large Program was established to complete the ESPaDOnS/Narval field sample, and to take the first steps towards observing massive stars in various open clusters and associations of different ages, to investigate the magnetic field evolution, and the impact of magnetic fields on stellar evolution.

The HARPSpol sample is divided into two components (SC and TC) to follow the same strategy as the Narval and ESPaDOnS LPs. The HARPSpol SC sample contains about 180 stars that include $\sim 110$ stars in seven clusters, and $\sim 70$ stars in the field of the Galaxy. The former have been selected from the Catalogue of Open Cluster Data (Kharchenko et al. 2005), while the latter have been chosen from the International Ultraviolet Explorer (IUE) data archive, but also from other catalogues or publications containing highly probable magnetic 
Table 1. Log of observations in 2011.

\begin{tabular}{|c|c|c|c|c|c|c|}
\hline $\begin{array}{l}\text { Date }(\mathrm{d} / \mathrm{m}) \\
\text { UT }\end{array}$ & $\begin{array}{c}\text { HJD } \\
(2455000+)\end{array}$ & $\begin{array}{l}t_{\exp } \# \\
(\mathrm{~s})\end{array}$ & $\# S / N$ & $\begin{array}{r}S / N \\
(\mathrm{LSD})\end{array}$ & $\begin{array}{l}B_{\ell} \\
(\mathrm{G})\end{array}$ & $P_{\mathrm{det}}$ \\
\hline \multicolumn{7}{|c|}{ HD 130807} \\
\hline $23 / 0$ & 704.724 & 40001 & 1500 & 4600 & $292 \pm 26$ & $1.00000 \mathrm{DD}$ \\
\hline $27 / 0$ & $6 \quad 708.754$ & 12001 & 1520 & 4800 & $-94 \pm 26$ & $1.00000 \mathrm{DD}$ \\
\hline 28/05 05:58 & $8 \quad 709.748$ & 60002 & 2630 & 5700 & $677 \pm 21$ & $1.00000 \mathrm{DD}$ \\
\hline \multicolumn{7}{|c|}{ HD 122451} \\
\hline 23/05 04:16 & 704.677 & 13405 & 51680 & 33200 & $-43 \pm 20^{a}$ & 1.000 \\
\hline $27 / 05$ 05:21 & 708.723 & 12002 & 203000 & 61800 & $-83 \pm 14^{a}$ & $1.00000 \mathrm{DD}$ \\
\hline 28/05 04:34 & 709.690 & 16801 & 141260 & 26000 & $-66 \pm 29^{a}$ & $0.98173 \mathrm{ND}$ \\
\hline \multicolumn{7}{|c|}{ HD 105382} \\
\hline $25 / 05$ & 706.555 & 32001 & 990 & 965 & $-622 \pm 26$ & $1.00000 \mathrm{DD}$ \\
\hline 26/05 01:59 & 707.582 & 40001 & 1900 & 884 & $-298 \pm 32$ & $1.00000 \mathrm{DD}$ \\
\hline 28/05 00:10 & D 709.506 & 48001 & 1920 & 8900 & $-406 \pm 32$ & $1.00000 \mathrm{DD}$ \\
\hline
\end{tabular}

Notes. Columns 1 and 2 give the date, Universal Time (UT) and Heliocentric Julian Date of the observations. Columns 3 and 4 give the total exposure time and the number of polarimetric sequences. Columns 5 and 6 give the peak $S / N$ per CCD pixel (at $\sim 501 \mathrm{~nm}$ for HD 130807 and at $\sim 518 \mathrm{~nm}$ for HD 122451 and HD 105382) in the spectra, and the $S / N$ per $1.4 \mathrm{~km} \mathrm{~s}^{-1}$ (for HD 130807 and HD 105382) and $4.2 \mathrm{~km} \mathrm{~s}^{-1}$ (for HD 122451) pixels in the LSD Stokes $V$ profiles. Columns 7-9 give the longitudinal magnetic field, the magnetic detection probability, and the detection type (see text). ${ }^{(a)}$ Those values are for HD 122451 B only.

stars, in accordance with the ESPaDOnS/Narval target selection (Wade et al. 2009).

This HARPSpol Large Program was allocated four separate runs over two years. During the first run in May 2011, 57 stars were observed including one magnetic calibrator and one TC target, the results which will be presented in a forthcoming paper. We here report on the first discoveries of magnetic fields in massive stars with HARPSpol in HD 130807 and HD 122451, and we confirm the magnetic field in HD 105382 that was previously detected with the low-resolution spectropolarimeter FORS 1 (Kochukhov \& Bagnulo 2006; Hubrig et al. 2006). Among the 55 SC stars observed during this run, those three stars are the only ones in which a magnetic field was detected. In Sect. 2 we present the observations and reduction techniques. In Sect. 3 we detail the HARPSpol results on each star, and discuss them in Sect. 4.

\section{Observations}

We used the HARPSpol polarimeter (Piskunov et al. 2011), combined with the HARPS spectrograph (Mayor et al. 2003), installed at the $3.6 \mathrm{~m}$ ESO telescope at La Silla Observatory (Chile), which yields spectra with resolving power $\lambda / \Delta \lambda$ of about 105000 and covers the 380-690 nm wavelength region. All spectra were recorded as sequences of four individual subexposures taken in different configurations of the polarimeter to yield a full circular polarisation analysis, as described by Donati et al. (1997). The data were reduced using the package "REDUCE" described by Piskunov \& Valenti (2002). After reduction, we obtained the intensity Stokes $I$ and the circular polarisation Stokes $V$ spectra of the stars, both normalised to the continuum. A null spectrum $(N)$ was also computed to diagnose spurious polarisation signatures, and to help to verify that the signatures in the Stokes $V$ spectrum are of stellar origin. The log of the observations is presented in Table 1.

To increase the effective signal-to-noise ratio $(S / N)$ of our data, we applied the least-squares deconvolution (LSD;
Donati et al. 1997) procedure, which uses tailored line-masks of appropriate temperature and gravity for each star. The masks were first computed using the Kurucz ATLAS 9 models of solar abundance (Kurucz 1993), with intrinsic line depths higher than 0.1 . We then excluded from these masks hydrogen Balmer lines, and lines whose Landé factor is unknown. Finally we modified the line depths to take into account the relative depth of the lines of the observed spectra. The resulting masks contain 394, 592, and 394 lines for HD 1030807, HD 122451 and HD 105382, respectively. The $S / N$ of the LSD Stokes $V$ profiles is about 10 times higher than the $S / N$ in the original spectra (Table 1).

To perform a reliable magnetic field diagnosis, we computed the detection probability inside the LSD $V$ profiles (as described in Donati et al. 1997). We consider that an observation displays a "definite detection" (DD) of Stokes $V$ Zeeman signature if the probability is higher than 0.99999, a "marginal detection" (MD) if it falls between 0.999 and 0.99999 , and a "null detection" (ND) otherwise (see Table 1). All observations of HD 130807 and HD 105382 display DD, while two DD and one ND were obtained for HD 122451. The LSD $I, V$, and $N$ profiles are plotted in Fig. 1. In almost all of our observations Zeeman signatures as broad as the $I$ profiles are clearly detected in the $V$ profiles, while the $N$ profiles are consistent with the noise. These results allow us to confidently affirm that magnetic fields are present on the surface of these stars.

We measured for each observation the line-of-sight component of the magnetic field averaged over the visible stellar surface (the so-called longitudinal magnetic field, or $B_{\ell}$ ) by integrating the $I$ and $V$ profiles over the ranges $[-50,60]$, $[-80,100]$, and $[-70,105] \mathrm{km} \mathrm{s}^{-1}$ for HD 130807, HD 122451, and HD 105382, respectively (as described by Alecian et al. 2009). The values are reported in Table 1.

\section{Results}

\subsection{HD 130807}

HD 130807 (o Lup) is a member of the Sco-Cen association (Kharchenko et al. 2005). A companion was detected at an angular distance varying from 0.07 to 0.14 arcsec (Perryman \& ESA 1997; McAlister et al. 1990). According to the angular separation, the light of both components entered the HARPSpol fibers during our observations of this target.

From a visual inspection we find that most of the spectrum of HD 130807 is consistent with a synthetic spectrum of a single star of effective temperature $T_{\text {eff }}=18000 \mathrm{~K}$, surface gravity $\log g=4.25$ (cgs), broadened by $v \sin i=25 \mathrm{~km} \mathrm{~s}^{-1}$, calculated using TLUSTY non-LTE atmosphere models and the SYNSPEC code (Hubeny 1988; Hubeny \& Lanz 1992). However, we observe that all He I lines are substantially weaker than the synthetic ones calculated with solar abundance (Fig. 2), while the $\mathrm{Si}$ II lines are considerably stronger. The $\mathrm{Si}, \mathrm{N}$, and $\mathrm{Fe}$ lines show variability in depth and shape on a timescale of $1 \mathrm{~d}$. These characteristics suggest that HD 130807 is an He-weak star with abundance spots on its surface (Jaschek \& Jaschek 1974).

Magnetic signatures are detected in almost all lines of the spectrum, similar to the LSD one (Fig. 1, left). Many additional lines are observed in the spectrum that can be caused by Fe II, Fe III, or Ti II enhancements. All these lines show Zeeman signatures similar to the others, with the same variations from one night to the other. They can therefore be attributed to the same star, rather than to a companion, and they are probably the result of the chemical peculiarities at the surface of the star.

A significant shift in radial velocity $\left(\sim 6 \mathrm{~km} \mathrm{~s}^{-1}\right)$ is detected in the strongest spectral lines including the Balmer lines, 

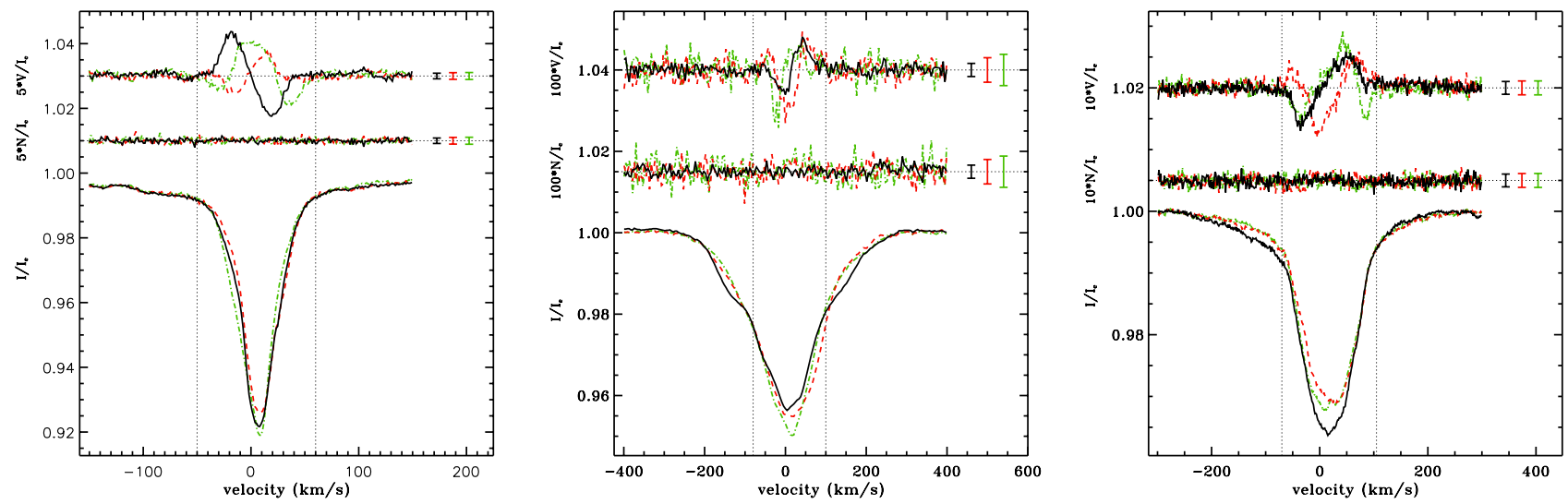

Fig. 1. LSD V (top), $N$ (middle), and I (bottom) profiles of HD 130807 (left), HD 122451 (middle), and HD 105382 (right). The mean error bars are plotted next to each profile. The $V$ and $N$ profiles were shifted and amplified for clarity. The dotted vertical lines indicate the integration ranges for the calculation of $B_{\ell}$. Left: full black line: 28/05, red-dashed line: 27/05, green dot-dashed line: 23/05. Middle: full black line: 27/05, red-dashed line: 23/05, green dot-dashed line: 28/05. Right: full black line: 25/05, red-dashed line: 28/05, green dot-dashed line: 26/05.

between May 22 and May 26-27. The maximum reported angular separation between both visual components implies a distance $\geq 17 \mathrm{AU}$, and therefore a period $\geq 27$ years. This radial velocity shift can therefore not be caused by the reported visual companion. A third companion very close to the primary could explain these variations, but more observations are required to fully understand all peculiarities observed in the spectrum.

The variations observed in the $V$ profiles over six days (Fig. 1, left) can be understood in terms of the oblique rotator (OR) model that consists of an inclined dipole placed inside a rotating star (Stibbs 1950). The rotational modulation of the shape of the $V$ profiles and of the $B_{\ell}$ values that vary from -94 to $677 \mathrm{G}$ (Table 1) suggest that the rotation period of the star should be between one and six days. More observations, well-sampled over the rotation period, are required to fully characterise the magnetic field and better constrain the period of HD 130807.

\section{2. $H D 122451$}

HD 122451 ( $\beta$ Cen) is a double-lined spectroscopic binary with components of similar effective temperatures $(25000 \mathrm{~K})$ and gravities ( $\log g=3.5, \operatorname{cgs})$, and a $\beta$ Cep-type pulsating primary. The system is highly eccentric $(e=0.835)$ and orbits with a period of 357 days (Ausseloos et al. 2002, 2006; Davis et al. 2005).

We obtained three observations of HD 122451 during three different nights. To avoid potential false magnetic detections caused by pulsations, we splitted each observation into many sequences of four spectra (Table 1), so that the exposure time for one sequence is much shorter than the pulsation period.

The spectra of HD 122451 clearly show two components of similar temperature, but different broadening, confirming the SB2 nature of the system. We adopt the same definition as Ausseloos et al. (2006) for the primary and secondary, i.e. as the broad-line and narrow-line components, respectively. To measure the $v \sin i$ of both stars, we performed a least-squares fit to several individual spectral lines with the sum of two functions calculated as the convolution of a Gaussian of instrumental width and a rotation function as described by Gray (1992) (see details of the fitting procedure used by Alecian et al. 2008). We find a $v \sin i$ of $190 \pm 20 \mathrm{~km} \mathrm{~s}^{-1}$ and $75 \pm 15 \mathrm{~km} \mathrm{~s}^{-1}$ for the primary and secondary, respectively. When compared with TLUSTY/SYNSPEC synthetic spectra our observations are consistent with $T_{\text {eff }}=25000 \mathrm{~K}$ and $\log g=3.5(\mathrm{cgs})$, which agrees

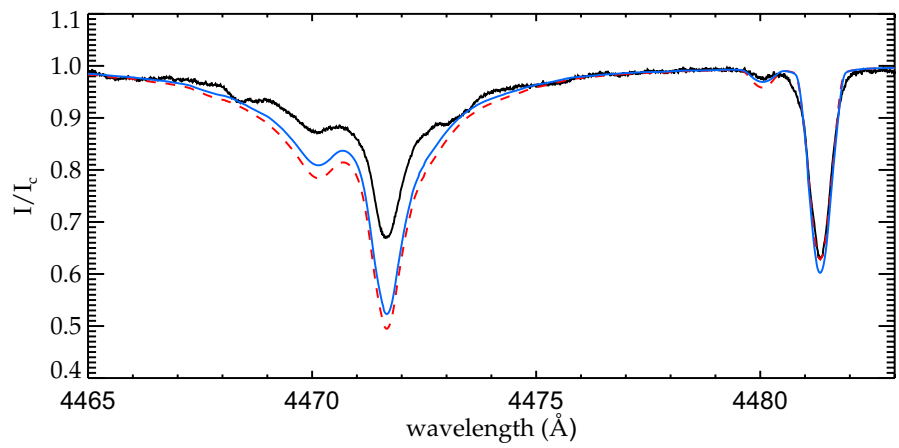

Fig. 2. Spectrum (full black) of HD 130807 plotted around He I $4471 \AA$ and Mg II $4481 \AA$. Synthetic spectra of $18000 \mathrm{~K}$ (dashed red) and $17000 \mathrm{~K}$ (dot-dot-dot-dashed blue) are overplotted.

with the work of Ausseloos et al. (2006). The spectral lines appear to be distorted and show rapid variations very likely caused by $\beta$ Cep-type pulsations. No obvious abundance peculiarity or manifestation of circumstellar matter is observed within the spectra.

In Fig. 1 (middle) we superimposed the LSD $I, V$, and $N$ profiles of our observations. According to the ephemeris of Ausseloos et al. (2006), the three observations are roughly at the same orbital phase $(\sim 0.5)$, and both components have similar radial velocities $\left(\sim 14\right.$ and $\sim 4 \mathrm{~km} \mathrm{~s}^{-1}$ for the primary and secondary respectively), which explains why it is difficult to distinguish both components in the profiles. The shape of the LSD I profile shows variations during the run, which can be understood in terms of radial pulsations in the primary, which would occasionally broaden the profile. As a result, both components can be clearly distinguished in the profile of May 27 (full black line in Fig. 1, middle), while it is less obvious in the other observations.

Zeeman signatures are detected in many individual spectral lines, which look similar to the LSD $V$ profiles. The signatures are as broad as the secondary profile, meaning that the magnetic field is detected only in the secondary component of the system. However, considering the faint Zeeman signatures in the secondary and the broad line shape of the primary, a magnetic field of the same strength as the secondary's could exist in the primary without being detected in our observations. To estimate the $B_{\ell}$ values of the secondary, we need to extract the $V$ and $I$ profiles 
of the secondary only from the profiles of the binary. Without any evidence of a magnetic field in the primary, we neglect its contribution to the $V$ profile, which we consider to be entirely from the secondary. But the $I$ profile needs to be corrected. With this aim, we first fitted the $I$ profile of the binary with the method described above for the individual spectral lines. Then we subtracted from the observed $I$ profile the fit of the primary. Finally we have measured $B_{\ell}$ using the corrected $I$ and the original $V$ profiles. The values are reported in Table 1.

\section{3. $H D 105382$}

HD 105382 (= HR 4618) is member of the Sco-Cen association (Kharchenko et al. 2005). Briquet et al. (2004) classified it as He-weak with He patches enhanced where $\mathrm{Si}$ is depleted, and derived a $T_{\text {eff }}$ of $17400 \pm 800 \mathrm{~K}$, a $\log g=4.18 \pm 0.20(\mathrm{cgs}), \mathrm{a}$ rotation period of $1.295 \pm 0.001 \mathrm{~d}$ and an inclination angle of the rotation axis to the line of sight $i=50 \pm 10^{\circ}$.

We observe strong variations in the spectral lines in our three spectra, mainly in He I, Si II and Fe III, which are caused by abundance spots on the stellar surface described by Briquet et al. (2004). Clear Zeeman signatures are detected in the metallic and Balmer lines, as well as in the LSD $V$ profiles (Fig. 1, right). The rotation phases of our observations, calculated with a rotation period of $1.295 \mathrm{~d}$, are very different $(0.35,0.14$, and 0.63$)$, and yet the $V$ profiles are all similarly negative (Table 1 ). According to the OR model, this implies that the magnetic obliquity angle (with respect to the rotation axis) cannot be very high $\left(|\beta|<40^{\circ}\right.$ if $i=50^{\circ}$ ), otherwise the positive magnetic pole would sometimes appear on the visible stellar hemisphere, creating a positive profile at least once during the run.

HD 105382 was independently discovered as magnetic by Kochukhov \& Bagnulo (2006) and Hubrig et al. (2006). Briquet et al. (2007) derived the longitudinal field from FORS 1 observations and found values ranging from $-923 \mathrm{G}$ to $840 \mathrm{G}$. Among their four values, the May 2004 observation $(840 \pm 58 \mathrm{G})$ is clearly inconsistent with our data because positive values are not expected. Bagnulo et al. (2011) very carefully re-reduced the same FORS 1 data and found values consistent with those of Briquet et al. (2007) except for that observation (for which they derived $B_{\ell}=-29 \pm 69 \mathrm{G}$ ).

We performed a least-squares sinusoidal fit to our $B_{\ell}$ values simultaneously with the Briquet et al. (2007) data and could find a solution only by removing the May 2004 datapoint. We also performed an independent fit using the re-reduced data of Bagnulo et al. (2011) and found a similar result. In both cases, the derived period is consistent with that of Briquet et al. (2004). The fitted $B_{\ell}$ values are very similar in both cases, varying from $-670 \mathrm{G}$ to $-20 \mathrm{G}$, and implying a magnetic obliquity of $\sim 38^{\circ}$ and a polar field strength of $\sim 2.3 \mathrm{kG}$, assuming a dipole field (Borra \& Landstreet 1980).

\section{Discussion}

We reported direct detections of magnetic fields in three hot B-type stars $(18000 \mathrm{~K}-25000 \mathrm{~K})$ among a sample of 55 stars in which we were searching for magnetic fields with HARPSpol. Two of them (HD 122451 and HD 130807) are completely new detections. For the other one - HD 105382 - this is the first direct detection of a Zeeman signature. One of the main MiMeS results is the systematic detection of chemical peculiarities at the surface of magnetic hot stars, and vice versa (e.g. Grunhut et al. 2011a). Among these three stars, two are unambiguously He-weak. The third one (HD 122451) belongs to a binary system with a $\beta$ Cep primary, which makes the interpretation of the spectrum and the detection of peculiarities inside spectral lines very difficult. More well-sampled observations over the orbital period of the system are required to first confirm a magnetic detection in only the secondary, and then separate the pulsation and chemical peculiarity effects.

The interplay between radiative and magnetic forces (Hunger \& Groote 1999) is usually assumed to be at the origin of the over- or underabundant He spots at the surface of hot magnetic B stars. These spots are very often correlated with the stellar magnetic fields (e.g. Veto 1990). In the case of HD 105382, Briquet et al. (2004) found a large He spot at a latitude of $60^{\circ}$. If our estimate of its magnetic obliquity is confirmed, this spot would be situated close to the South magnetic pole, demonstrating once more the importance of magnetic fields in the formation of chemical spots.

More observations of these magnetic stars are planned within the HARPSpol large program to perform detailed mapping of their magnetic fields, and better confront the models and theories of magnetic massive stars.

Acknowledgements. We wish to thank the Programme National de Physique Stellaire (PNPS) for their support. J.H.G. and G.A.W. acknowledges support from NSERC. M.B. acknowledges the Fund for Scientific Research - Flanders for a grant for a long stay abroad; she is a F.R.S.-FNRS Postdoctoral Researcher. RHDT acknowledges support from NSF grant AST-0908688. This research has made use of the SIMBAD database and the VizieR catalogue access tool, operated at CDS, Strasbourg (France), of INES data from the IUE satellite and of NASAs Astrophysics Data System.

\section{References}

Alecian, E., Catala, C., Wade, G. A., et al. 2008, MNRAS, 385, 391 Alecian, E., Wade, G. A., Catala, C., et al. 2009, MNRAS, 400, 354 Ausseloos, M., Aerts, C., Uytterhoeven, K., et al. 2002, A\&A, 384, 209 Ausseloos, M., Aerts, C., Lefever, K., et al. 2006, A\&A, 455, 259

Bagnulo, S., Landstreet, J. D., Fossati, L., \& Kochukhov, O. 2011, A\&A, accepted

Borra, E. F., \& Landstreet, J. D. 1980, ApJS, 42, 421

Briquet, M., Aerts, C., Lüftinger, T., et al. 2004, A\&A, 413, 273

Briquet, M., Hubrig, S., Schöller, M., \& De Cat, P. 2007, Astron. Nachr., 328, 41

Davis, J., Mendez, A., Seneta, E. B., et al. 2005, MNRAS, 356, 1362

Donati, J.-F., Semel, M., \& Carter, B. D. 1997, MNRAS, 291, 658

Gray, D. F. 1992, The observation and analysis of stellar photospheres., ed. D. F. Gray

Grunhut, J. H., Wade, G. A., Marcolino, W. L. F., et al. 2009, MNRAS, 400, L94

Grunhut, J. H., Rivinius, T., Wade, G. A., et al. 2011a, MNRAS, in press [arXiv: 1109.3157]

Grunhut, J. H., Wade, G. A., \& the MiMeS Collaboration 2011b [arXiv: 1108.2673]

Hubeny, I. 1988, Comput. Phys. Comm., 52, 103

Hubeny, I., \& Lanz, T. 1992, A\&A, 262, 501

Hubrig, S., North, P., Schöller, M., \& Mathys, G. 2006, Astron. Nachr., 327, 289 Hunger, K., \& Groote, D. 1999, A\&A, 351, 554

Jaschek, M., \& Jaschek, C. 1974, Vistas Astron., 16, 131

Kharchenko, N. V., Piskunov, A. E., Röser, S., Schilbach, E., \& Scholz, R.-D. 2005, A\&A, 438, 1163

Kochukhov, O., \& Bagnulo, S. 2006, A\&A, 450, 763

Kurucz, R. 1993, Opacities for Stellar Atmospheres: [-3.5], [-4.0], [-4.5]. Kurucz CD-ROM No. 7. Cambridge, Mass.: Smithsonian Astrophysical Observatory, 7

Mayor, M., Pepe, F., Queloz, D., et al. 2003, The Messenger, 114, 20

McAlister, H., Hartkopf, W. I., \& Franz, O. G. 1990, AJ, 99, 965

Oksala, M. E., Wade, G. A., Townsend, R. H. D., et al. 2011, MNRAS, in press [arXiv: 1109.0328]

Perryman, M. A. C., \& ESA, eds. 1997, The HIPPARCOS and TYCHO catalogues, ESA-SP, 1200

Piskunov, N., Snik, F., Dolgopolov, A., et al. 2011, The Messenger, 143, 7

Piskunov, N. E., \& Valenti, J. A. 2002, A\&A, 385, 1095

Stibbs, D. W. N. 1950, MNRAS, 110, 395

Veto, B. 1990, Ap\&SS, 174, 111

Wade, G. A., Alecian, E., Bohlender, D. A., et al. 2009, in IAU Symp., 259, 333 